\title{
EDITORIAL
}

\section{Is it time to consider ethnicity in treatment guidelines?}

A ny good researcher studying cardiovascular disease with the aid of one of more mouse models will tell you that you cannot compare a C57BL/6 mouse with a SVJ129, BALB/C, or FVB mouse, even if it has been bred and raised in exactly the same environment in the same animal-care facility. They may all be mice, and it may appear that the only major difference is their coat color, but numerous studies have demonstrated differences in their 'normal' cardiovascular functions, which can have considerable effect on the findings of a study. For example, 'normal' FVB mice have been shown to have increased left ventricular ejection fraction, fractional shortening, and heart rate compared with 'normal' SVJ129, BALB/C, and C57BL/6 mice (Shah, A. P. et al. Cardiovasc. Res. 87, $683-693 ; 2010)$ and so should not be used as wild-type control animals for comparison with SVJ129, BALB/C, or C57BL/6 mice genetically modified to have cardiovascular disease. Furthermore, infarct rupture after myocardial infarction occurs more frequently in C57BL/6 mice than in their BALB/C counterparts (van den Borne, S. W. et al. Cardiovasc. Res. 84, 273-282; 2009), and 129Xi/SvJ and 129 SvImJ mice demonstrate almost normal cardiac function during in vivo acute hypoxia, whereas C57BL/6J mice exhibit massive cardiac decompensation in the same conditions (Barnabei, M. S. et al. Physiol. Genomics 42A, 103-113; 2010).

We use mouse models of disease because they are considered to be relatively good models of human disorders; it seems reasonable, therefore, to use different mouse strains as an analogy for different human ethnic groups. Indeed, in line with what we know about mouse experiments, we acknowledge the importance of similar ethnic spreads in comparisons of placebo-treated and experimental groups of patients in clinical trials.

So why isn't our knowledge of ethnic differences reflected in human disease guidelines? The issue is not as straightforward as it seems and specific reasons for these differences should be investigated more thoroughly before we consider undertaking this kind of approach.

A major problem with having distinct guidelines for different ethnic groups is that we currently have incomplete knowledge as to whether many of the observed differences between ethnic groups result from social or physiological factors. A retrospective single-center study (Collins, S. D. et al. Circulation 122, 1085-1090; 2010) published online in late August demonstrated that black ethnicity was a predictor of definite drug-eluting-stent thrombosis, and that this relationship was independent of socioeconomic status. Of the multiple variables

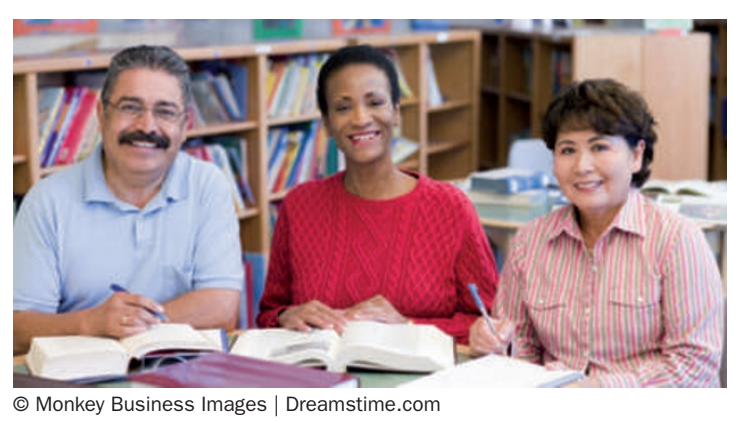

assessed, hypertension, diabetes mellitus, chronic renal insufficiency, and congestive heart failure were more prevalent in black patients than in nonblack individuals. However, multivariable analysis demonstrated that black race was an independent predictor of late stent thrombosis and that the aforementioned comorbidities were not. In addition to ruling out the effect of socioeconomic status, the authors ruled out difference in access to care as being a likely explanation for the study's finding, since all patients had undergone percutaneous coronary intervention and adherence to clopidogrel was actually better among black patients than among nonblack individuals. However, the authors' description of access to care was specific to the percutaneous coronary intervention and they admit that incidence of stent thrombosis could have been affected by confounding variables not represented in their model. These potential confounding factors could result from reduced access to general health care or to health education. On the other hand, the prognostic difference observed in this study might well be explained by ethnicity-related differences in one or more genetic or proteomic factors.

The findings of a meta-analysis (Shah, T. et al. Circ. Cardiovasc. Genet. 3, 436-444; 2010) published online in September instigated debate on whether a single cut-off point for C-reactive protein (CRP) across multiple ethnic groups was appropriate for the determination of statin prescription. In this study, mean CRP levels differed between patients grouped by ethnicity and these differences were only partially explained by the well-established risk factors of BMI, systolic blood pressure, and smoking status. Again, a question that remains is whether these differences could have been explained by additional risk-factor differences between the study groups. Furthermore, even if CRP levels truly are in part determined by physiological differences between ethnic groups, should these differences result in different cut-off values for CRP in treatment guidelines? As Paul Ridker
Bryony M. Mearns is the Chief Editor of Nature Reviews Cardiology.

Competing interests The author declares no competing interests 
(Brigham and Women's Hospital, Boston, MA, USA) points out, "vascular event rates also vary between ethnic groups and ... this variation tracks with CRP levels".

Another problem with having distinct recommended treatment targets for different ethnic groups was highlighted in the 2009 ACCF/AHA heart failure guidelines: "Race is an imprecise concept that has become a social and political construct, with more limited biological significance" (2005 Writing Committee Members et al. Circulation 119, e391-e479; 2009). Indeed, unlike the various mouse strains used in research, genome sequencing studies have demonstrated huge genetic variation among people grouped as having the same ethnicity. For example, a study of the genomes of 5 individuals, each from a different tribe in South Africa-all of whom would be grouped together as having 'black' ethnicity, and who come from a relatively small region of the worlddemonstrated more variation than would be seen from a comparison of an Asian genome with that of a European (Schuster, S. C. et al. Nature 463, 943-947; 2010).

A further problem is that many individuals cannot easily be classified into one specific ethnic group. If the aforementioned within-ethnic-group genetic variation is ignored and specific guidelines for specific ethnic groups are generated, which guidelines would we use for individuals born from parents of different ethnic backgrounds? At the end of the last century, the issue of having an appropriate baseline (control) resulted in researchers around the world scrambling to back-cross their mixedstrain mouse models to generate 'genetically pure' strains, so that they had an available baseline to which they could accurately compare their disease models and, therefore, generate valid conclusions. By contrast, with the numerous multicultural communities found in most regions around the globe, mixed-race individuals are extremely common in today's world and will certainly become more prevalent. Guidelines for specific ethnic groups, therefore, would not be applicable to a vast proportion of the population.
No-one denies that information on a patient's ethnicity can be useful when considering a patient's likelihood of developing a specific disease or experiencing a particular clinical outcome. Social differences in access to care and/or health education (including information on diet, smoking, and the importance of exercise) probably account for many of the clinical differences currently attributed to race, although Collins et al. argue that difference in access to care is an unlikely explanation for the ethnicity-related differences they observed in stent thrombosis. Immediate efforts should, therefore, be focussed on ensuring equal access to care and health education across the different ethnic groups within our communities, rather than on generating race-specific treatment guidelines on the basis of incomplete information.

Detailed knowledge of genetic and proteomic differences between different ethnic groups is currently lacking, and it is virtually impossible for researchers to account for every social or environmental variable that might be responsible for observed ethnic differences in clinical outcomes-the 'real world' is vastly different from the highly controlled environment of an animal-care facility in this respect. Until the observed ethnicity-related differences in clinical outcome can be attributed to specific physiological differences, it seems premature and somewhat short-sighted to start writing treatment guidelines with specific goals that vary on the basis of ethnicity. With the rapidly expanding quantity of data generated by genome-wide association studies and proteomic investigations, it would perhaps be more appropriate to work towards varying treatment decisions on the basis of patients' specific genetic and proteomic backgrounds-rather than the broad and often inaccurate classification of ethnicity-in addition to currently established environmental and comorbidity risk factors. This strategy would provide appropriate treatment targets for all individuals, including those of mixed race.

doi:10.1038/nrcardio.2010.181 